ROCZNIKI PSYCHOLOGICZNE/ANNALS OF PSYCHOLOGY

2019, XXII, 1, 91-102

DOI: http://dx.doi.org/10.18290/rpsych.2019.22.1-6

MONIKA WRÓBEL ${ }^{\mathrm{a}}$

MICHAŁ OLSZANOWSKI ${ }^{\mathrm{b}}$

${ }^{a}$ University of Lodz, Institute of Psychology, Poland

${ }^{\mathrm{b}}$ SWPS University of Social Sciences and Humanities in Warsaw, Poland

\title{
EMOTIONAL REACTIONS \\ TO DYNAMIC MORPHED FACIAL EXPRESSIONS: A NEW METHOD TO INDUCE EMOTIONAL CONTAGION
}

\begin{abstract}
In the current study, we tested the utility of a new method developed to study emotional contagion (i.e., the transfer of emotional states between people). Inspired by studies on emotional mimicry a process that has been postulated as one of the main mechanisms leading to emotional contagion, we created a set of videos showing morphed facial expressions of happiness, sadness, and anger. Following exposure to each video, participants rated their emotions. Our findings demonstrated that the videos evoked congruent emotions in viewers, thereby supporting the notion that dynamic morphed facial expressions may be effective "emotionally contagious" stimuli. Additionally, in line with the previous studies and classic theories of emotional contagion, the displays of anger evoked a complementary reaction of fear.
\end{abstract}

Keywords: emotional contagion; basic emotions; facial expressions of emotion; emotion-eliciting stimuli; morphing.

\section{INTRODUCTION}

For many years, researchers have studied the tendency for people to "catch" the emotions of others. The process, often referred to as emotional contagion, involves various mechanisms by which the emotional expression of one person

Corresponding author: MONIKA WRÓBEL - Institute of Psychology, University of Lodz, ul. Smugowa 10/12, 91-433 Lódź, Poland; e-mail: monika.wrobel@uni.lodz.pl

This research was supported by the Polish National Science Centre Grants 2017/26/E/ HS6/00725 (to M. Wróbel) and 2016/21/HS6/01179 (to M. Olszanowski). The data that support the findings of this study are openly available in the Open Science Framework at https://osf.io/zea6d. 
- the sender - evokes a congruent emotional state in another person - the receiver (Hatfield, Cacioppo, \& Rapson, 1994). Two prime examples of such mechanisms are emotional mimicry (i.e., the automatic imitation of the sender's emotional display by the receiver), and social appraisal (i.e., a more reflective process by which the receiver integrates information derived from the sender's emotional expression into his/her own thoughts and feelings about the situation) (Parkinson, 2011; Wróbel \& Imbir, 2019).

Emotional contagion may be studied by means of a number of techniques. For instance, participants may be exposed to a confederate who has been trained to express target emotions (e.g., Barsade, 2002). Their emotional reactions may also be studied in "real-world" contexts (e.g., leader-employee or teacher-student relationships; Houser \& Waldbuesser, 2017; Johnson, 2008). These methods, however, are challenging when investigating multiple emotions, because it might be difficult to create comparable experimental conditions (Kuhlen \& Brennan, 2013). Therefore, the majority of experimental studies on emotional contagion rely on more standardized techniques, such as pictures or videos of emotional displays (e.g., Lundqvist \& Dimberg, 1995; Papousek, Schulter, \& Lang, 2009). Here, we take a closer look at these techniques and analyze their limitations. We also compare these methods to the methods used in studies on emotional mimicry. Building on this comparative analysis, we propose a new type of visual stimuli that can be used in emotional contagion research and report the results of a study that tested the utility of this new method.

\section{Visual stimuli used in emotional contagion research}

Early research on emotional contagion relied on videos in which senders spontaneously recalled the happiest or the saddest events in their lives (Doherty, Orimoto, Singelis, Hatfield, \& Hebb, 1995; Hsee, Hatfield, Carlson, \& Chemtob, 1990). For instance, Hsee et al. (1990) used two videos that lasted about 1:30 min and presented a man who described either his surprise birthday party or his grandfather's funeral. His spontaneous facial, postural, and vocal emotional expressions conveyed feelings corresponding to the recalled events.

Although those early videos facilitated laboratory research on emotional contagion, they had a considerable disadvantage. Specifically, it was impossible to determine whether participants reacted to the sender's emotional displays or to the recalled events. To address this limitation, Doherty (1997) developed three videos that differed in terms of the emotionally expressive behavior of the sender 
(happy, sad, and neutral) and in terms of length (2:50, 3:55, and 3:05 min, respectively) but were identical in terms of message content. The video-taped woman, whose mood was induced with hypnotic suggestions, recited a scripted fictitious message about the purpose of the study. The script, however, made the videos unsuitable for other research (e.g., in which the scripted message interfered with the cover story). To circumvent this problem, Wróbel (2016) developed two 1-minute videos without sound. Here, prior to the recording, a male sender was primed with either happy or sad photographs and then, for the recording, he was instructed to look into the camera and think about the happiest or the saddest events in his life.

Importantly, in all of the aforementioned studies, the video-taped senders expressed target emotions in a recognizable way (as indicated by independent judges' ratings) and these emotions spread to viewers (as indicated by participants' self-reported feelings). This demonstrates that 1-3-minute videos of spontaneous emotional behavior are effective "emotionally contagious" stimuli. A closer analysis, however, suggests that the utility of such "long" videos is limited by two factors. First, they typically show either male or female senders and therefore the gender effects they might produce are difficult to control for. Second, given that happiness and sadness are easier to induce and sustain in laboratory settings than other emotions (Siedlecka \& Denson, 2018), "long" videos usually present only these two expressions. Therefore, it is difficult to determine whether the effects of such videos are specific to the induced emotions or limited to the valence dimension.

Papousek et al. (2009) addressed the second of the above issues by creating a set of videos showing posed emotional displays. The set, referred to as the Emotionally Contagious Films (ECOFs), consists of five silent videos (1:20 min each) that show a female actress displaying sadness, fear, anger, happiness, and neutral expression. The films are silent but the actress's emotional expression is extremely intense (e.g., intense weeping, hearty laugh, or aggressive behavior). However, as no equivalent set of videos showing a male sender was developed, the ECOFs do not solve the first of aforementioned problems. We believe that this gap may be filled by using the methods inspired by studies on emotional mimicry, that is, as already mentioned, a process considered one of the basic mechanisms of emotional contagion. 


\section{Visual stimuli used in emotional mimicry research}

Researchers studying emotional mimicry often rely on pictures or video clips of basic emotional expressions (e.g., Hess \& Blairy, 2001; van der Schalk et al., 2011; Wingenbach, Brosnan, Pfaltz, Plichta, \& Ashwin, 2018). Participants are thus exposed to either static or dynamic emotional displays, while their facial activity is measured with electromyography.

Compared to the videos used in emotional contagion research, video clips used to study emotional mimicry are much shorter (from 1 to $5 \mathrm{~s}$ ) and typically present posed emotional displays. Therefore, they are easier to create and validate than the "long" videos used in emotional contagion studies (i.e., a person in a video does not have to sustain his/her emotional expression for several seconds or minutes). As a result, the development of stimuli showing both female and male senders displaying different emotions is less challenging. Moreover, "short" videos enable researchers to compare the effects of multiple emotional expressions, because the displays such stimuli present are relatively equivalent in terms of the senders' emotional behavior. For instance, while a "long" video showing an angry sender may differ considerably from a "long" video showing a sad sender (anger is expressed more intensively and represented by a wider repertoire of nonverbal signals than sadness), "short" video clips of these two emotional expressions are quite comparable in terms of the senders' facial actions. One more advantage of the videos used in emotional mimicry research is that, due to their brevity, they may be presented in a randomized sequence, which makes studying the effects for multiple emotions easier (e.g., Sachisthal, Sauter, \& Fischer, 2016; Wingenbach et al., 2018).

The question remains whether such short videos are sufficient to produce emotional contagion, which, as already mentioned, involves not only emotional mimicry but also more reflective (and therefore slower) processes such as social appraisal. A partial answer to this question can be provided by a few studies on emotional mimicry that involved additional assessment of participants' self-reported emotions (Hess \& Blairy, 2001; van der Schalk et al., 2011; Sachisthal et al., 2016). In general, these studies demonstrate that participants report feeling emotions that correspond to those of the senders, but these effects are usually rather weak, vary across different emotions, and are accompanied or even dominated by the induction of complementary emotional states (e.g., fear in response to angry displays). For instance, Hess and Blairy (2001) found clear evidence for happiness and sadness contagion, but not for anger and disgust contagion (angry 
faces evoked repulsion/fear, whereas disgusted faces did not evoke any specific emotion). It is possible that the lack of evidence for contagion in the case of some emotional displays results from the fact that participants exposed to videos that are too short do not have enough time to observe the senders and therefore find it difficult to feel and absorb the target emotions. We think that this problem can be solved by bridging the gap between emotional contagion and emotional mimicry research - that is, by using videos of basic emotions that last longer than a couple of seconds. We explore this possibility in the current study.

\section{The present study}

The aim of this research was to test the utility of a new method developed to study emotional contagion. Specifically, by integrating emotional contagion and emotional mimicry research paradigms, we created a set of 35-second videos based on displays of basic emotions shown by male and female senders.

In creating the videos, we applied morphing, that is, a computer technique that generates smooth transitions between images. By gradually changing one image into another, morphing allows for good control of the onset, duration, and intensity of emotional displays. Importantly, this technique has already been applied in studies on emotional mimicry (e.g., Achaibou, Pourtois, Schwartz, \& Vuilleumier, 2008), but the dynamic emotional expressions used in those studies lasted no longer than 1:30 s, thereby posing the same problems as other "short" videos used in emotional mimicry research. Here, as already mentioned, we decided to create longer videos. At the same time, given that our stimuli did not present spontaneous emotional behavior but morphed facial displays of basic emotions, we assumed that, if too long, they might be boring to participants. Therefore, we decided that 35 seconds would provide a good compromise between the two paradigms.

\section{METHOD}

\section{Participants}

A power analysis, using the $\mathrm{G}^{*}$ Power 3 software (Faul, Erdfelder, Lang, \& Buchner, 2007), indicated that a sample size of 36 would provide power of .95 to detect a medium-sized effect in a repeated measures ANOVA with four levels. 
Therefore, we recruited 40 participants ( 24 females; $M_{\text {age }}=21.62, S D=2.08$ ). All of them were undergraduate psychology students who participated in the study in exchange for course credit and provided written informed consent.

\section{Stimuli}

We used videos of happiness, anger, and sadness, because these three emotions are most often studied in emotional contagion and emotional mimicry research (see Wróbel, 2016, for a review). To create the videos, we used pictures of two male and two female models taken from the Warsaw Set of Emotional Facial Expression Pictures (WSEFEP; Olszanowski et al., 2015). The images were selected on the basis of the facial action unit (AU) scores provided for each picture. Specifically, we relied on AU12 (lip corner puller) for happiness, AU15 (lip corner depressor) for sadness, and AU4 (brow lowerer) for anger. In total, we created 12 videos (4 senders x 3 emotional expressions).

Using FantaMorph 5.0 software, we synthesized dynamic emotional displays gradually changing from a neutral expression to a happy, sad, or angry expression of the same face identity (see Figure 1). Each video began with a 2 -second static frame showing a neutral face that morphed into an emotional one within $3 \mathrm{~s}$. Once the expression reached the apex (i.e., full emotional display), it morphed again (within the next 3 s) into a blended expression (50\% neutral and 50\% happy/sad/angry). Next, it reached the apex again (after $3 \mathrm{~s}$ ). These steps were repeated four times. The last frame showed a static image of the blended expression.

The videos were then analyzed with iMotions 7.0 software equipped with AFFDEX algorithms (Stöckli, Schulte-Mecklenbeck, Borer, \& Samson, 2018). The aim of this analysis was to test whether the transformations described above had not obscured the emotional expressions of happiness, sadness, and anger. The analysis demonstrated that the activity of critical action units (i.e., AU12, AU15, and AU4, respectively) was still visible, thereby confirming that the morphed faces displayed the intended emotions. 


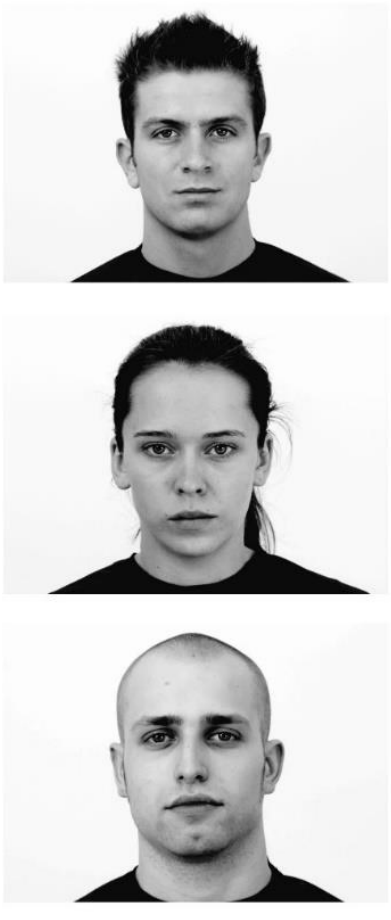

a
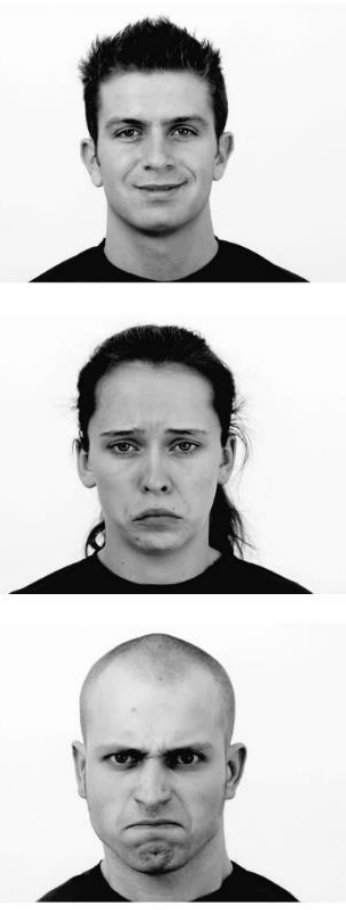

b

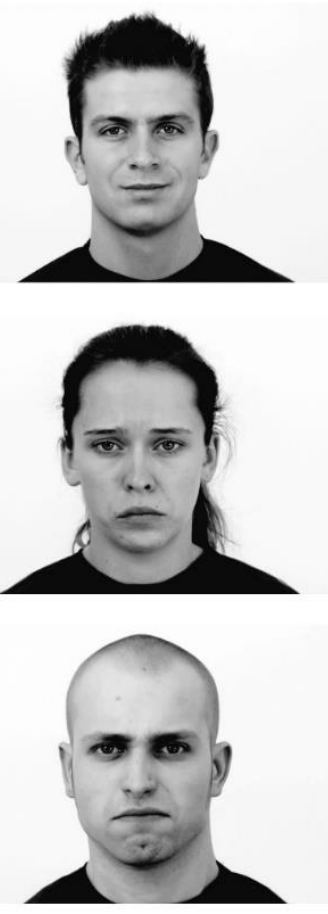

C

Figure 1. Sample frames showing neutral emotional display (a), apex (b), and a blended emotional expression (c).

\section{Measures}

To measure participants' self-reported emotions, we used a modified Polish version of the Differential Emotion Scale (DES; Izard, Doherty, Bloxom, \& Kotsch, 1974). The scale consists of twelve adjectives describing four emotions: happiness (happy, cheerful, delighted), sadness (sad, downhearted, blue), anger (angry, irritated, $\mathrm{mad}$ ), and fear (anxious, fearful, tense). We measured fear in addition to the three target emotions because, as already mentioned, angry displays may also evoke complementary emotional reactions. Participants rated the extent to which they felt each state when watching each video using a scale ranging from 1 (not at all) to 7 (extremely). In the current study, Cronbach's $\alpha$ s ranged from .87 to .97 . 


\section{Procedure}

Participants viewed 12 video clips presented on a computer screen. To avoid any biased answers, we led them to believe that they would be making judgments about other people. Each trial was preceded with a 2 -second fixation cross and followed by the self-reported emotions scale. To lend credence to the cover story and minimize the risk of interference between the trials, participants answered two filler questions about the likeability and competence of the presented person. Additionally, to reduce learning effects, we presented both the videos and the adjectives used to measure participants' emotions in a random order.

\section{RESULTS}

To examine whether the emotional states reported by participants were consistent with the emotions displayed by the senders, we conducted a series of repeated measures ANOVAs with four levels of self-reported emotions (happiness $v s$ sadness $v s$ fear $v s$ anger) separately for the three emotions displayed by the senders. The analyses showed significant effects for happiness, $F(3,37)=$ $=234.68, p<.001, \eta_{\mathrm{p}}{ }^{2}=.86$, sadness, $F(3,37)=42.96, p<.001, \eta_{\mathrm{p}}{ }^{2}=.52$, and anger, $F(3,37)=12.62, p<.001, \eta_{\mathrm{p}}{ }^{2}=.24$ (see Figure 2$)$.

Planned contrast comparisons $(p<.05$; contrast weights: $+1,-1,0,0)$ demonstrated that the videos evoked target emotions in the viewers. Specifically, after being exposed to happy displays, participants reported significantly more happiness $(M=5.02, S D=1.18)$ than sadness $(M=1.36, S D=0.62)$, anger $(M=1.39, S D=0.46)$, and fear $(M=1.43, S D=0.58)$. Exposure to sad displays resulted in higher levels of sadness $(M=4.25, S D=1.61)$, as compared to the levels of happiness $(M=1.89, S D=1.04)$, anger $(M=1.84, S D=0.78)$, and fear $(M=2.42, S D=1.00)$. Finally, exposure to the senders' anger resulted in feeling significantly more anger $(M=3.32, S D=1.56)$ than happiness $(M=1.83$, $S D=1.19)$ and sadness $(M=2.55, S D=1.21)$. The levels of fear $(M=3.43$, $S D=1.48$ ) induced by angry displays, however, were not significantly different from the levels of self-reported anger. ${ }^{1}$

\footnotetext{
${ }^{1}$ We also conducted a series of two-way repeated measures ANOVAs with participants' self-reported emotions (happiness $v s$ sadness $v s$ fear $v s$ anger) and the senders' sex (male $v s$ female) as within-subjects factors to see if the effects of the senders' displays were moderated by their sex. The analyses demonstrated that: (1) happy female senders evoked slightly higher happiness $(M=$ $=5.32, S D=1.01)$ than happy male senders $(M=4.72, S D=1.54 ;(2)$ sad female senders evoked slightly higher sadness $(M=4.45, S D=1.75)$ than sad male senders $(M=4.04, S D=1.69)$; and
} 


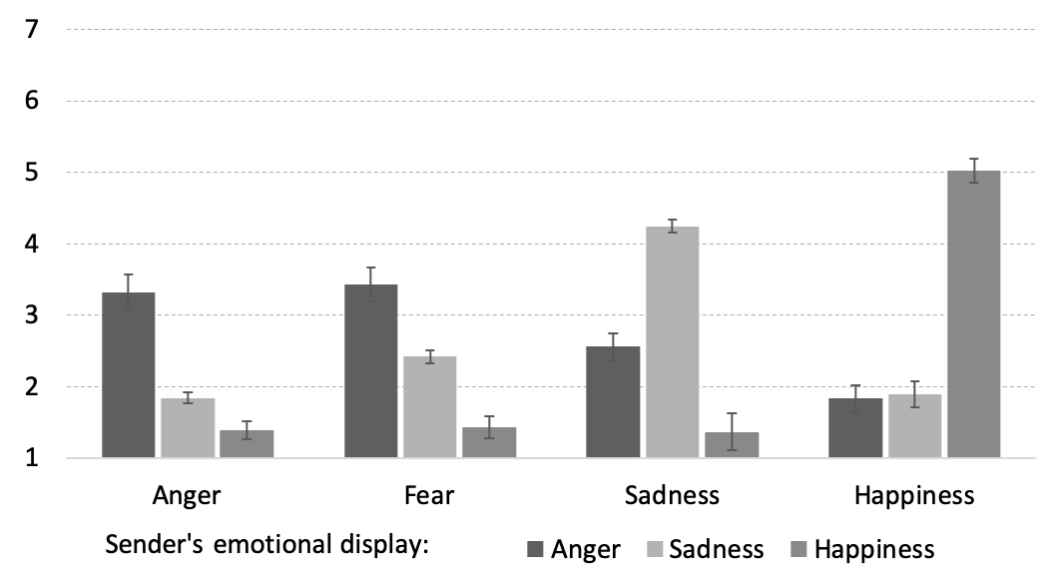

Figure 2. Participants' self-reported emotions following exposure to the senders' emotional displays. Higher scores indicate more intense self-reported emotions. Error bars represent standard errors of the mean.

\section{DISCUSSION}

In the current study, we tested the utility of a new method developed to investigate emotional contagion. Based on the comparison between studies on emotional contagion and studies on emotional mimicry, we created twelve 35 -second videos presenting happy, sad, and angry senders. Our results demonstrated that the new videos were effective in inducing emotional contagion, which may be attributed to two facts. First, as the videos were based on pictures of basic emotions taken from a well-validated database (Olszanowski et al., 2015), the expressions were genuine and unambiguous, which may have facilitated emotional contagion. Second, the videos were probably long enough to provide the participants with sufficient time to feel the emotions displayed by the senders.

It is important to note that, in line with the previous studies (Hess \& Blairy, 2001; Lundqvist \& Dimber, 1995) and with the classic model of emotional contagion (Hatfield et al., 1994), exposure to angry senders resulted not only in

(3) angry male senders evoked slightly higher fear $(M=3.72, S D=1.56)$ than angry female senders $(M=3.14, S D=1.54)$. The interpretation of these findings, however, is obscured by the fact that the stimuli included only two female and two male faces. It is therefore not clear whether the aforementioned differences were caused by the sex or individual facial features of the senders (e.g., physical attractiveness; see Królewiak \& Wróbel, 2017). 
a convergent emotional state (that is, contagion) but also in a complementary reaction of fear. This suggests that exposure to anger triggered not only "mirroring" of the senders' expression (which was manifested by a convergent emotional reaction), but also appraisal mechanisms. Specifically, participants may have interpreted angry faces as threatening, which in turn evoked fear. This pattern of results is in line with the theories holding that emotional contagion and mimicry are not simple "reflex-like" phenomena but may be controlled by top-down processes (e.g., receivers' interpretations) (Fischer \& Hess, 2017; Wróbel \& Imbir, 2019).

At the same time, we should note that what our method adds to existing techniques is that anger induced by angry senders was not dominated by fear but the levels of these two emotional states were comparable. This finding seems crucial here for two reasons. First, anger in general is very difficult to induce in laboratory settings, especially when videos are used (Lobbestael, Arntz, \& Wiers, 2008; Siedlecka \& Denson, 2018). This suggests that our method provides an alternative route to anger induction. Second, although previous studies have indicated that anger is hardly ever imitated because it is a non-affiliative emotional expression (see Fischer \& Hess, 2017, for a review), our findings suggest that exposure to anger may result in anger contagion when the video is long enough.

Some limitations of this study should also be acknowledged. First, as the method we propose is new, we focused on only three emotions that appear in emotional contagion and mimicry literature most frequently. Thus, the question remains whether other basic emotions could also be induced in a similar way. We therefore hope that future research will address this issue by examining the applicability of our method to other emotional displays. Second, when describing their emotional reactions, participants rated the levels of only four emotions. This was related to the fact that we used a within-subject design and thus, similarly to studies on emotional mimicry, restricted the assessment to critical emotional states. This enabled us to keep participants attentive and involved in the study (Sachistal et al., 2016), but limited our conclusions to the four emotions we measured. At the same time, though, it is possible that the videos evoked some additional emotional reactions. Thus, caution is still warranted when interpreting the current findings.

Overall, our study is the first to demonstrate that the videos showing morphed dynamic expressions of basic emotions may induce emotional contagion. By addressing the limitations of the "long" videos used in previous studies, our method allows for studying the effects of multiple emotions displayed by both 
male and female senders. This seems to be a promising avenue for future research on the transfer of emotional states between people.

\section{REFERENCES}

Achaibou, A., Pourtois, P., Schwartz, S., \& Vuilleumier, P. (2008). Simultaneous recording of EEG and facial muscle reactions during spontaneous emotional mimicry. Neuropsychologia, 46, 1104-1113.

Barsade, S. G. (2002). The ripple effect: Emotional contagion and its influence on group behavior. Administrative Science Quarterly, 47, 644-675.

Doherty, R. W. (1997). The Emotional Contagion Scale: A measure of individual differences. Journal of Nonverbal Behavior, 21, 131-154.

Doherty, R. W., Orimoto, L., Singelis, T. M., Hatfield, E., \& Hebb, J. (1995). Emotional contagion: Gender and occupational differences. Psychology of Women Quarterly, 19, 355-371.

Faul, F., Erdfelder, E., Lang, A.-G., \& Buchner, A. (2007). G*Power 3: A flexible statistical power analysis program for the social, behavioral, and biomedical sciences. Behavior Research Methods, 39, 175-191.

Fischer, A., \& Hess, U. (2017). Mimicking emotions. Current Opinion in Psychology, 17, 151-155.

Hatfield, E., Cacioppo, J. T., \& Rapson, L. R. (1994). Emotional contagion. Cambridge: Cambridge University Press.

Hess, U., \& Blairy, S. (2001). Facial mimicry and emotional contagion to dynamic emotional facial expressions and their influence on decoding accuracy. International Journal of Psychophysiology, 40, 129-141.

Houser, M. L., \& Waldbuesser, C. (2017). Emotional contagion in the classroom: The impact of teacher satisfaction and confirmation on perceptions of student nonverbal classroom behavior. College Teaching, 65, 1-8.

Hsee, Ch. K., Hatfield, E., Carlson, J. G., \& Chemtob, C. (1990). The effect of power on susceptibility to emotional contagion. Cognition and Emotion, 4, 327-340.

Izard, C. E., Dougherty, F. E., Bloxom, B. M., \& Kotsch, N. E. (1974). The Differential Emotions Scale: A method of measuring the subjective experience of discrete emotions. Unpublished manuscript, Vanderbilt University, Nashville, TN.

Johnson, S. K. (2008). I second that emotion: Effects of emotional contagion and affect at work on leader and follower outcomes. The Leadership Quarterly, 19, 1-19.

Królewiak, K., \& Wróbel, M. (2017). Czy uśmiech ładnych ludzi jest bardziej zaraźliwy? Atrakcyjność fizyczna jako moderator zarażania afektywnego [Are smiles of attractive people more contagious? Physical attractiveness moderates affect contagion]. Psychologia Spoleczna, 12, 397-404.

Kuhlen, A. K., \& Brennan, S. E. (2013). Language in dialogue: When confederates might be hazardous to your data. Psychonomic Bulletin \& Review, 20, 54-72.

Lobbestael, J., Arntz, A., \& Wiers, R. W. (2008). How to push someone's buttons: A comparison of four anger-induction methods. Cognition and Emotion, 22, 353-373.

Lundqvist, L.-O., \& Dimberg, U. (1995). Facial expressions are contagious. Journal of Psychophysiology, 9, 203-211. 
Olszanowski, M., Pochwatko, G., Kuklinski, K., Scibor-Rylski, M., Lewinski, P., \& Ohme, R. (2015). Warsaw Set of Emotional Facial Expression Pictures: A validation study of facial display photographs. Frontiers in Psychology, 5, 1516. DOI: 10.3389/fpsyg.2014.01516.

Papousek, I., Schulter G., \& Lang, B. (2009). Effects of emotionally contagious films on changes in hemisphere-specific cognitive performance. Emotion, 51, 1018-1022.

Parkinson, B. (2011). Interpersonal emotion transfer: Contagion and social appraisal. Social and Personality Psychology Compass, 5, 428-439.

Sachisthal, M. S. M., Sauter, D. A., \& Fischer, A. H. (2016). Mimicry of ingroup and outgroup emotional expressions. Comprehensive Results in Social Psychology, 1, 86-105.

Siedlecka, E., \& Denson, T. F. (2018). Experimental methods for inducing basic emotions: A qualitative review. Emotion Review, 11(1), 87-97. DOI: 10.1177/1754073917749016

Stöckli, S., Schulte-Mecklenbeck, M., Borer, S., \& Samson, A. C. (2018). Facial expression analysis with AFFDEX and FACET: A validation study. Behavior Research Methods, 50, 1446-1460.

Van der Schalk, J., Fischer, A., Doosje, B., Wigboldus, D., Hawk, S., Rotteveel, M., \& Hess, U. (2011). Convergent and divergent responses to emotional displays of ingroup and outgroup. Emotion, 11, 286-298.

Wingenbach, T. S. H., Brosnan, M., Pfaltz, M. C., Plichta, M. M., \& Ashwin, C. (2018). Incongruence between observers' and observed facial muscle activation reduces recognition of emotional facial expressions from video stimuli. Frontiers in Psychology, 9, 864. DOI: 10.3389/fpsyg.2018.00864

Wróbel, M. (2016). Zarażanie afektywne. O procesie transferu emocji i nastroju między ludźmi [Affect contagion: On the transfer of emotions and moods between people]. Warszawa, Poland: Wydawnictwo Naukowe PWN.

Wróbel, M., Imbir, K. (2019). Broadening the perspective on emotional contagion and emotional mimicry: The correction hypothesis. Perspectives on Psychological Science, 14, 437-451. 